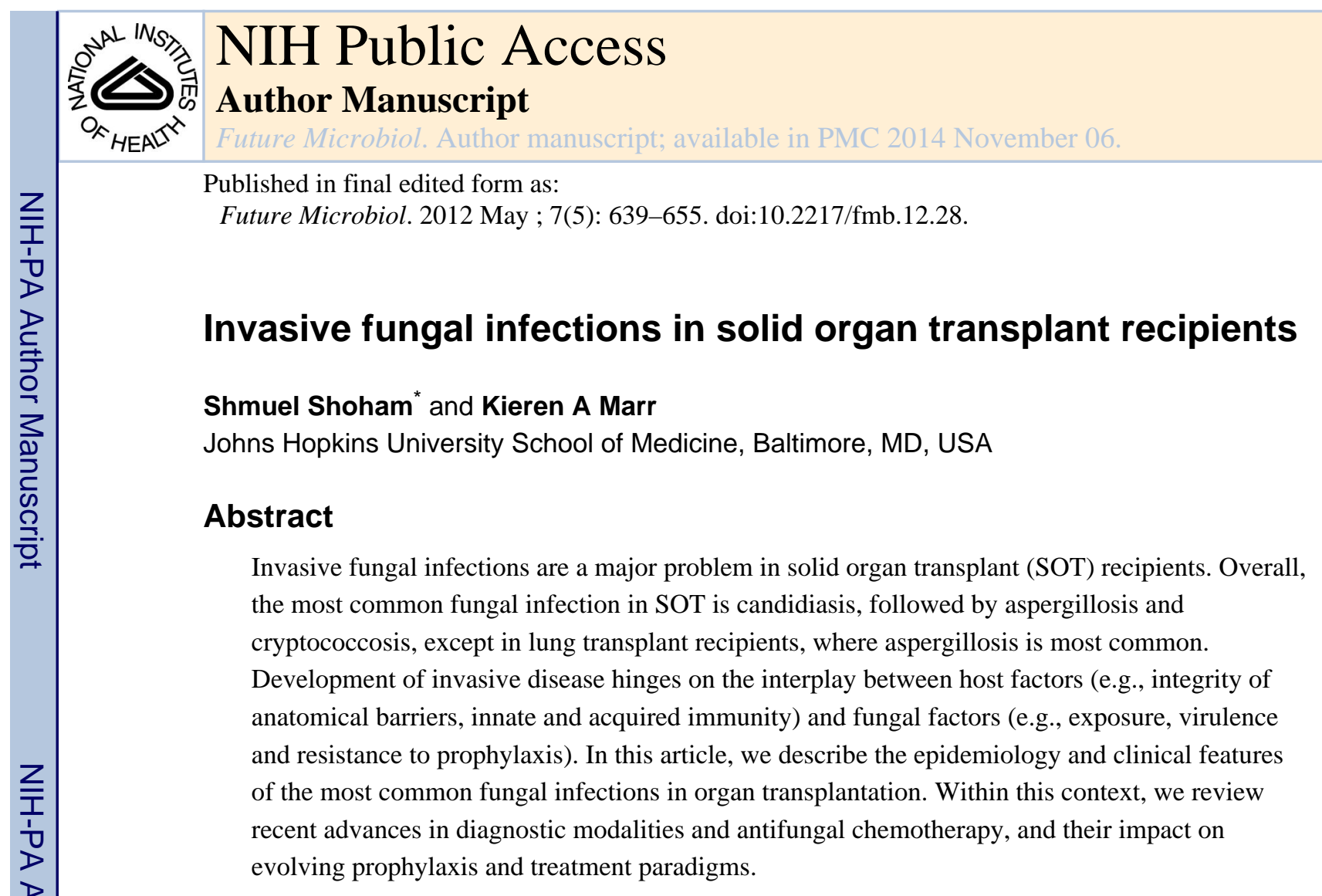

\title{
Keywords
}

antifungal; aspergillosis; candidiasis; cryptococcosis; fungal; galactomannan; kidney transplant; liver transplant; lung transplant; transplant

\begin{abstract}
Improvements in therapeutic and diagnostic options are providing clinicians with unprecedented tools to evaluate, manage and prevent invasive fungal infection in solid organ transplant (SOT) recipients. Despite these advances, invasive fungal infections continue to be a major cause of morbidity and mortality in this population. Recent studies have shed new light on the epidemiology of invasive fungal infections in SOT recipients [1-3]. The overall cumulative incidence during the first year after transplantation is approximately $3 \%$, although this varies depending on the type of organ transplanted [1]. However, the risk of infection, particularly due to inhaled fungi, persists for many years after transplant. Current epidemiological trends indicate a shift towards later infections. The consequences of fungal infection can be dire and include long hospitalizations, allograft damage and high mortality rates. Data from 15 centers involved in a prospective cohort study of invasive fungal infections in SOT recipients indicate that mortality at 12 months is approximately $40 \%$ for aspergillosis, $34 \%$ for candidiasis and $27 \%$ for cryptococcosis [1]. In this article, we review the epidemiology, clinical presentations, diagnostic techniques and therapeutic options for the most common invasive fungal infection in SOT recipients.
\end{abstract}

(C) 2012 Future Medicine Ltd

*Author for correspondence: Tel.: +1 4106146431 Fax: +1 4106148518 sshoham1@jhmi.edu.

Financial \& competing interests disclosure

The authors have no other relevant affiliations or financial involvement with any organization or entity with a financial interest in or financial conflict with the subject matter or materials discussed in the manuscript apart from those disclosed.

No writing assistance was utilized in the production of this manuscript. 


\section{General epidemiology}

Host and environmental factors are critically important determinants in the epidemiology of fungal infections in transplantation. The highest risk is in small bowel (11.6\%) and lung $(8.6 \%)$ transplants, followed by liver $(4.7 \%)$, heart $(4.0 \%)$, pancreas $(3.4 \%)$ and kidney (1.3\%) [1]. Factors that impact the risk for developing an invasive fungal infection include the patient's environmental exposure and/or colonization with pathogenic fungi, use of antifungal prophylaxis, as well as the net state of immuno-suppression. The latter refers to the combined impact of immune suppressing factors, including antirejection therapies, breaches in mucocutaneous barriers, leukopenia, comorbid conditions (e.g., malnutrition, cirrhosis, diabetes mellitus and hypogammaglobulinemia) and chronic viral infections (e.g., CMV, HCV, HBV and HIV) [4].

The net state of immunosuppression varies widely depending on the type of transplant received. The interplay between host and environmental factors and the impact of antifungal prophylaxis strategies is more relevant to development of specific fungal infections than number of days after transplantation. For example, the median time to onset of invasive candidiasis ranges from several weeks to months in lung and liver transplant recipients, to over 2 years in kidney recipients [2]. Similarly, the median time to invasive aspergillosis is $<6$ months in liver transplant recipients, but occurs much later in kidney, heart and lung transplant recipients. The latter onset of invasive aspergillosis in lung transplant recipients is likely influenced by widespread use of mold-active prophylaxis in that population. Finally, cryptococcosis tends to occur between 2 and 5 years post-transplant, but can be observed much earlier in cases of donor transmission or heavy environmental exposure [2].

Candidiasis is the most common invasive fungal infection in SOT recipients and accounts for 50-60\% infections [1]. Candida species, particularly Candida albicans, are frequent colonizers of the human gastrointestinal, respiratory and reproductive tracts, and the skin. The majority of invasive candidiasis is from an endogenous source - usually the skin or gut. Aspergillosis is the next most common infection, accounting for $20-25 \%$ of fungal infections. In lung transplant recipients, aspergillosis is the most common infection [2]. Infection may be due to reactivation of a previously quiescent process such as colonization or subclinical infection, or from de novo infection following inhalation of this ubiquitous mold after transplantation. The remaining infections are due to Cryptococcus species (6$7 \%$ ), the endemic fungi (5\%) and many other rare and emerging mycoses [1,2]. Reactivation of latent infection is a major mode of disease in cryptococcosis, histoplasmosis and coccidioidomycosis. Chronic carriage of fungi pretransplantation is a particular problem in unilateral lung transplant recipients and cystic fibrosis patients. In these patients, the retained lung and/or abnormal upper respiratory tract and sinuses can act as reservoirs for potentially pathogenic fungi that can then progress to cause invasive disease.

Donor-derived infections are an increasingly recognized mode of transmission [5]. Transplanted organs may act as reservoirs for potentially pathogenic fungi. Transmission of an array of pathogenic fungi including Aspergillus species, Candida species, Histoplasma capsulatum, Cryptococcus neoformans, Coccidioides immitis and Scedosporium apiospermum has been reported [6-19]. Active fungal disease in the donor is a 
contraindication to transplantation, but infection may be latent and unknown to the donor and transplant team. This is particularly relevant to endemic mycoses and cryptococcosis, which are often present in dormant forms. Sometimes, unexplained symptoms in the donor are only understood in retrospect once the recipients develop infection. Differentiating donor-derived infection from reactivation of latent infection within the recipient may be difficult. Transplant centers should immediately notify the organ procurement agency if there is suspicion for transmission of a fungal pathogen, as it may impact care of the other recipients from a common donor. As yet, there are no uniform recommendations for donor screening for endemic mycoses [16].

\section{Specific infections}

\section{Candidiasis}

SOT recipients are at increased risk for invasive candidiasis, the most common fungal infection in this population. In a recent study, the overall annual estimated incidence of candidemia in SOT recipients was significantly higher than in other hospitalized patients (3 vs 0.21 per 1000 admissions; $\mathrm{p}$ < 0.001) [20]. As in other patients, $C$. albicans is the most common cause of invasive candidiasis and accounts for approximately $50 \%$ of cases. Candida parapsilosis is often associated with infections of indwelling medical devices and has emerged as an important pathogen in SOT. Candida glabrata ( 30\%) and Candida krusei $(\sim 5 \%)$ are important pathogens in SOT recipients, especially in those who have received prior antifungal therapy $[2,21]$.

Invasive candidiasis is nearly always preceded by colonization [22]. The risk for invasive disease is related to intensity of colonization, which can be increased by exposure to broadspectrum antibiotics, corticosteroid use, diabetes mellitus, prolonged stay in an intensive care unit (ICU) and urinary catheterization [23-25]. Although colonization is generally a prerequisite for infection, even heavy colonization does not invariably lead to invasive disease. The development of invasive candidiasis depends upon the virulence of the organism and impairment of critical host defenses. Examples of the latter include disruptions of mucosal and cutaneous barriers with chronic indwelling intravascular devices and prolonged operative time, and abnormalities in neutrophil function and number as can be caused by high-dose immunosuppressants [26].

Transplant recipients, particularly of abdominal organs, have multiple risk factors for invasive candidiasis. These include receipt of broad-spectrum antibiotics, presence of central venous catheters, abdominal surgery and corticosteroid use. Complicated operative courses, renal dys-function and dialysis, receipt of parenteral nutrition and hyperglycemia all increase the risk for invasive candidiasis in SOT recipients [27-29]. Iron overload is emerging as a risk for invasive fungal infection. For example, in liver transplant recipients, stainable iron in the hepatic explant has been associated with post-transplantation candidiasis [30]. The type of anastomosis can also impact the risk of infection. In that regard, liver transplant recipients with a choledochojejunostomy are at higher risk for candidiasis compared with those having a choledocho-choledocho anastomosis. Similarly, pancreas transplant recipients with enteric drainage have higher rates of candidiasis compared with those with bladder drainage [31]. Bowel transplant recipients are at 
particularly high risk for invasive candidiasis, especially when there is rejection or dysfunction of the enteric graft, when the anastomosis is disrupted and in instances of enhanced immunosuppression, abdominal reoperation or multivisceral transplantation [3133].

Transmission of Candida species during the transplantation process has been documented. Candida may contaminate the organ while the donor is still alive, or during the procurement, processing and transplantation process. In cases of renal transplantation, the infection may involve the wound site, urinary tract, renal parenchyma or renal vasculature and can lead to organ loss $[17,34,35]$. Organ preservation fluid may also become contaminated with Candida species and serve as a conduit for transmission. Avoiding use of organs from patients with active candidiasis and routinely monitoring culture of preservation media are important preventative strategies [36].

Most cases of invasive candidiasis involve the bloodstream and/or the abdomen. In a recent ana lysis of 266 SOT patients with invasive candidiasis, 141 (53.0\%) had candidemia and 98 (36.8\%) had abdominal infection [2]. Bloodstream candidiasis may originate from translocation of organisms across damaged intestinal mucosa or occur at the site of a central venous catheter. Intra-abdominal candidiasis (e.g., peritoneal, perinephric and biliary infections) mainly affects recipients of abdominal organs [37,38]. Postoperative intraabdominal infections are a frequent complication of liver transplantation. Candida species are present in approximately $10 \%$ of such infections [39]. Bilomas, which are infected hepatic fluid collections, are a potentially devastating complication of liver transplantation. They are associated with high rates of death and need for retransplantation. Candida species can account for approximately $25 \%$ of such infections [40,41]. Compared with other pathogens, candidal bilomas are less likely to respond to nonsurgical therapy and are associated with increased mortality [41].

Invasive candidiasis continues to be associated with high rates of morbidity, mortality and excess medical costs [20]. Central venous catheters generally need to be removed in cases of bloodstream candidiasis. However, such devices may be difficult to replace and catheter management should be individualized for each patient. Moreover, host characteristics, including severity of illness, older age and immune status, are likely more important factors than early catheter removal [42]. Overall mortality at 12 weeks is in the $20-40 \%$ range and may be particularly poor in heart transplant recipients. Risks for poor outcomes in SOT patients with invasive candidiasis include dialysis dependence, mechanical ventilation and neutropenia, infections due to non-albicans Candida species and infections that break through antifungal prophylaxis $[2,29]$. Organ loss may ensue when infection involves the renal vasculature, pancreas and biliary tree.

The diagnosis can be straightforward, as when Candida species are identified from a normally sterile site such as bloodstream, intra-abdominal fluid or abscess material. However, interpretation of culture results from nonsterile sites can be challenging. Isolation of Candida species from stool, skin surfaces, drains, respiratory secretions and urine does not necessarily indicate infection, but may be a clue to patients at higher risk for developing an infection. Moreover, the sensitivity of bloodstream cultures for detecting Candida is 
suboptimal and many patients with disseminated disease can have negative blood cultures [43]. Identifying Candida to the species level is important because of interspecies variability in pathogenic potential and susceptibility to anti-fungal agents. Germ tube formation in the presence of serum is a rapid test that is suggestive (but not diagnostic) of $C$. albicans. Assays commonly used for speciation include appearance of colony growth on selective media (CHROMagar ${ }^{\mathrm{TM}}$ Candida), metabolic testing (e.g., $\mathrm{API}^{\circledR}{ }^{\circledR}$ Yeast) and fluorescent in situ hybridization (PNA FISH ${ }^{\circledR}$ ).

Use of nonculture diagnostic tests such as PCR and 1,3- $\beta$-D-glucan (BG) assays may provide another avenue for detecting invasive candidiasis. Performance characteristics of Candida PCR were recently evaluated in a meta-ana lysis that included nearly 5000 patients, 963 of whom had proven/probable or possible infection. In that ana lysis, sensitivity and specificity were in the 90-100\% range and use of whole-blood samples, rRNA or P450 gene targets and a PCR detection limit of $\leq 10 \mathrm{CFU} / \mathrm{ml}$ were associated with improved test performance [44]. The role of Candida PCR is as yet unclear in SOT recipients, but it appears to be an attractive option. Another approach is BG testing, and this assay may be further along in development. Patients with a range of pathogenic fungi including Aspergillus, Candida and Pneumocystis may have detectable serum BG levels. The sensitivity and specificity of the BG assay have been estimated to be in the $75-85 \%$ range [45]. However, the true sensitivity and specificity of these assays are not known in SOT recipients. Estimates of test performance characteristics are further hampered by the lack of a 'gold standard', as even blood cultures can be insensitive for detecting candidemia. Falsely elevated BG levels ( $\ 60$ $\mathrm{pg} / \mathrm{ml}$ ) have been described in association with multiple conditions common to transplant recipients, including receipt of hemodialysis, infections due to Pseudomonas aeruginosa, exposure to gauze and receipt of amoxicillin/clavulanate [46-49]. Although promising, the role of this assay for diagnosing candidiasis remains unclear in SOT recipients.

Nevertheless, difficulties in diagnosing invasive candidiasis in a timely manner and the potential for early diagnosis and treatment to impact outcomes is necessitating a move toward preemptive treatment strategies that rely on nonculture-based tools such as serum BG levels.

Treatment of invasive candidiasis in SOT recipients is similar to that of other patients. In general, initial therapy should be with an echinocandin (caspofungin, micafungin or anidulafungin) [50]. An echinocandin should also be considered as part of empiric therapy for sepsis of unclear etiology in SOT recipients, particularly in the early postoperative period and in recipients of abdominal organs. C. parapsilosis and Candida guilliermondii demonstrate less in vitro susceptibility to the echinocandins, and may need to be treated with alternative agents.Patients who are stable, not neutropenic and have not had recent azole exposure may be candidates for initial therapy with fluconazole. Independent risk factors for nonsusceptibility to fluconazole in SOT recipients include prior exposure to that drug, female gender, post-transplant diabetes mellitus and receipt of ganciclovir. Lipid formulations of amphotericin B (AmB) or voriconazole can be used as alternative agents, but are associated with increased toxicity. As indicated above, when feasible, removal of central venous catheters is strongly recommended [31]. Duration of therapy should be at 
least 2 weeks after clearance of the bloodstream cultures and resolution of symptoms attributable to candidemia.

Once patients have stabilized, they can be considered for conversion to fluconazole or voriconazole based upon the species of Candida isolated and susceptibility profile. Both agents have the advantage that they can be given by mouth, but voriconazole is associated with increased toxicity and drug interactions. The latter's use for the treatment of candidiasis should be reserved for cases of fluconazole resistance with maintained voriconazole susceptibility. As in other populations, fluconazole resistance in C. albicans, C. tropicalis and $C$. parapsilosis isolates is low $(\sim 1 \%)$ in transplant recipients [21]. A substantial proportion of $C$. glabrata isolates and all $C$. krusei have reduced susceptibility to fluconazole. Voriconazole may be an option for these patients. The spectrum of activity of posaconazole with respect to Candida is generally similar to that of voriconazole [21,50]. However, the role of posaconazole in invasive candidiasis is not clear.

Invasive candidiasis is less common in lung transplants. When infection does occur, it is usually as bloodstream infection and is much less common than pleural space infection or other forms of invasive disease [51]. Candida species are frequently found in respiratory samples from lung transplant recipients and donors [52]. These are rarely clinically relevant, although transmission of Candida from a donor lung has been reported, with resultant pulmonary, peritoneal and bloodstream involvement in the recipient [14].

Candidal airway or lung infection (as opposed to colonization) is rare in the current era of anti-fungal prophylaxis. Infection of the anastomosis, before it has had time to heal, may occur in the early postoperative setting. In such cases, the infection can lead to failure of the anastomosis, parenchymal lung infection and even mediastinitis [51,53-56]. Another type of infection can occur in patients with artificial bronchial stents. These devices are used to prevent airway closure in cases of airway or anastomotic narrowing, bronchomalacia and bronchial stenosis or stricture. The stents have a tendency to become plugged with debris and secretions, which can then serve as a site of airway and/or lung infection with a variety of fungi, including Candida and Aspergillus species [55]. Evidence of candidal tracheobronchitis is based on visual inspection and histologic confirmation and are usually supported by a positive culture from an appropriate specimen [31]. Therapy is not routinely commenenced solely on the basis of a positive respiratory culture; however, some authors recommend antifungal treatment for patients with Candida from bronchoalveolar samples and otherwise unexplained decline in respiratory function [55].

The clinical importance of candiduria in kidney transplant recipients is not completely understood. Candiduria is common with an estimated incidence of 3-11\% in kidney transplant recipients $[57,58]$. The infection is almost always ascending in nature from a periurethral source or from urinary catheterization. Much less frequently, urinary candidiasis occurs as a consequence of disseminated diseases with secondary seeding of the kidney and urinary tract. Risk factors for candiduria include female gender, admission to ICU, antibiotic use, the presence of an indwelling bladder catheter, diabetes mellitus, neurogenic bladder and malnutrition [58]. Candiduria is associated with decreased survival rate in transplant recipients, but this is thought to be due to greater severity of illness and comorbidities. Not 
all patients with candiduria need to be treated beyond removal of urinary catheters $[31,58]$.

Patients who have symptoms of infection, renal involvement, neutropenia and those undergoing urological procedures should be treated with an antifungal agent.

For those patients in whom treatment of candiduria is indicated and who have infection due to fluconazole-susceptible Candida spp., treatment with that antifungal agent is fairly straightforward. Treatment options for cystitis due to fluconazole resistant organisms are more complicated. Neither voriconazole nor the echinocandins penetrate into the urine well. Lipid formulations of AmB and flucytosine may be options, but these are associated with substantial risks for toxicity and many isolates develop resistance to flucytosine. For patients with fluconazole-resistant pyelonephritis, or hematogenous renal candidiasis, an echinocandin may be a reasonable option, as success typically depends on therapeutic serum and tissue (as opposed to urine) concentrations [31,59].

Antifungal prophylaxis against candidiasis has a role in some liver, pancreas and small bowel transplant recipients. Liver transplant recipients with multiple risk factors for candidiasis ( $\searrow$ wo of the following risk factors: prolonged or repeat operation, retransplantation, renal failure, high trans-fusion requirement, choledochojejunostomy and Candida colonization in the perioperative period) should receive prophylaxis. Pancreas transplant recipients with enteric drainage, vascular thrombosis and post-perfusion pancreatitis and nearly all small bowel transplant recipients should also be considered for prophylaxis [31]. Owing to the potentially devastating impact of invasive candidiasis in terms of morbidity and mortality in immunocompromised patients, prophylaxis strategies are increasingly employed beyond the above groups. Many critically ill transplant recipients are now placed on agents active against Candida species during their stays in the ICU. Risk stratification using intensity of Candida colonization, clinical characteristics and local infection patterns are employed by many institutions as a guide to prophylaxis [60-62]. The ideal agent is unclear, but fluconazole, an echinocandin and AmB products are reasonable options [31,63-66]. The latter two may also protect against aspergillosis, which can sometimes complicate high-risk liver transplants. The length of prophylaxis is not known, but is generally 4 weeks or longer, depending upon time to healing of anastomosis and resolution of risk factors.

\section{Aspergillosis}

Exposure is almost exclusively with inhalation of Aspergillus conidia from an environmental source. As such, infection nearly always involves the respiratory tract and/or sinuses. The most common infecting species is Aspergillus fumigatus. Infections due to Aspergillus flavus, Aspergillus niger and Aspergillus terreus are less common [67,68]. Clinically apparent infection may be from progression of a previously quiescent subclinical process or due to infection after transplantation. The latter can sometime occur in clusters due to a common source, such as building construction [69,70]. Nosocomial transmission leading to colonization and even infection has been described [71]. In one notable case, debridement and dressing of wounds from a patient with aspergillosis resulted in aerosolization of spores and airborne person-to-person transmission in a transplant ICU [72]. 
Transmission of Aspergillus via transplantation may occur directly from the organ or as a consequence of contamination of organ preservation fluid by airborne spores. Transmission to recipients has been documented following organ donation from patients who had themselves received organ transplantation shortly before they died $[9,13]$. In one of these cases, culture of a tracheal aspirate from the donor obtained at the time of organ donation eventually grew A. fumigatus [13]. Unusual sites of infection such as the urinary tract, graft anastomosis and heart valve are suggestive of donor-derived infection [9]. Prompt treatment of other organ recipients from the source patient can help avert their development of invasive aspergillosis [9]. Antifungal prophylaxis should also be considered in patients receiving organs from donors with positive Aspergillus respiratory cultures.

Key factors in development and timing of invasive aspergillosis are the recipient's net state of immunosuppression, intensity of exposure and the type of organ transplanted. Overall, invasive aspergillosis is more likely to occur in patients with renal failure, hemodialysis, repeated bacterial infections, leukopenia, CMV disease and in those requiring high levels of immunosuppression or retransplantation [73-76]. Highly immunosuppressed and chronically ill patients who then become critically ill can be at high risk for early-onset aspergillosis. Typical of such patients are liver transplant recipients, who require emergent transplantation, renal replacement therapy, large amounts of blood products and prolonged ICU stays. Conversely, patients who develop later-onset aspergillosis tend to be older, are heavily immunosuppressed and have chronically impaired graft function [73]. In the current era, where use of prophylactic and pre-emptive mold-active antifungal agents is increasingly used in the highest risk patients, the onset of invasive aspergillosis may be delayed by many months.

The highest risk for aspergillosis infection occurs in lung transplant recipients. In these patients, invasive aspergillosis accounts for nearly $50 \%$ of fungal disease [1]. In addition to the risk factors described above, chronic exposure of the transplanted lung to the environment and abnormal anatomical and physiological function of the transplanted and, if still present, the native lung, all predispose to infection. Lung transplant recipients frequently have Aspergillus colonization of the transplanted and/or native lung and upper respiratory tract. Colonization may lead to infection and has also been associated with development of bronchiolitis obliterans syndrome post-lung transplantation [77]. Additional risk factors for invasive aspergillosis in this population include airway ischemia, hypogammaglobulinemia (especially when IgG levels are less than $400 \mathrm{mg} / \mathrm{dl}$ ), concomitant CMV pneumonia, cystic fibrosis, placement of a bronchial stent and single lung transplantation [78-80].

Clinical manifestations may range from asymptomatic colonization to tracheobronchitis, invasive pulmonary aspergillosis, empyema and disseminated disease [81]. The presentation may be subtle with chronic cough, fever or malaise. Aspergillus tracheobronchitis may cause airway obstruction, ulcerations and pseudomembrane formation. As described above, airway stents have a tendency to become plugged and can be a site of airway and/or lung infection with a variety of fungi, including Aspergillus. Sites of infection beyond the respiratory tract include the skeletal system, thyroid, skin and CNS $[82,83]$. These are frequently due to dissemination from a primary respiratory tract site. Liver transplant recipients may be at 
particularly high risk for disseminated disease and CNS involvement compared with other transplant recipients [73]. Urinary tract aspergillosis has been reported in association with transmission through the graft. Such infections may also involve the vascular anastomosis site and almost invariably lead to loss of the transplant $[9,13]$.

Diagnosis of invasive aspergillosis can be challenging and frequently require histological evidence for infection and culture. Specimens (e.g., smears and tissue) can be stained with Gomori's Methenamine Silver and periodic acid-Schiff or with fluorescent dyes such as Calcofluor white [84]. Immunohistochemistry techniques are another promising diagnostic modality, but are not in widespread use as of yet. Culture remains an important modality for identification of Aspergillus to the species level and to improve sensitivity in cases of negative fungal staining. Identifying the species of Aspergillus can provide important clues to antifungal susceptibility and pathogenic potential of the organism identified. Aspergillus tends to grow well on routine media, but yields can be increased with fungal media such as Sabouraud's dextrose agar. Specimens that are obtained from nonsterile sites should be cultured in the presence of antibiotics to reduce bacterial growth.

Potentially infected specimens may require invasive procedures (e.g., bronchoscopy and/or lung biopsy) in order to be obtained, and typical staining and culture techniques are often insensitive. Radiographic approaches to early diagnosis have become increasingly important. However, radiographic characteristics of pulmonary infection are variable and can include nodules or masses. On CT these may be associated with surrounding groundglass opacity, central low density, central air cavity or air bronchograms [85]. In contradistinction to patients with hematological malignancies, halo signs are rarely observed in SOT patients with invasive aspergillosis. In cases of tracheobronchitis without lung parenchyma involvement, radiography may fail to reveal lesions that are visualized by bronchoscopy.

Major advances in nonculture techniques are allowing earlier diagnosis and reducing the need for invasive procedures. These include serum BG, nucleic acid assays (e.g., qualitative and quantitative PCR) of blood, bronchoalveolar lavage fluid and tissue and galactomannan testing. Of these, serum galactomannan, an assay that is now commercially available, is the furthest along. The utility of this test in SOT recipients for ruling out aspergillosis is limited. A recent meta-analysis of studies in SOT recipients reported that the sensitivity and specificity of the test were 22 and $84 \%$, respectively [86]. Application of this assay to bronchoalveolar lavage fluid facilitates the diagnosis of invasive pulmonary aspergillosis in organ transplant recipients [87]. Using a GM index of $\searrow 0.5-1.0$, the specificity exceeds $90 \%$ and sensitivity has ranged from 60-90\% [88-91]. False-positive results have been associated with certain antimicrobials (e.g., piperacillin/tazobactam and ampicillin) but this has not been consistent $[92,93]$. False positives can also be observed in lung transplant recipients, likely reflecting frequent filamentous fungi colonizing the airways in this population [87].

Invasive aspergillosis can be a catastrophic complication in SOT recipients and is associated with high rates of graft loss and death. Data from 15 centers involved in a prospective cohort study indicate that mortality at 12 months for SOT recipients with aspergillosis exceeds $50 \%$ [1]. However, mortality varies according to clinical presentation and host factors. For 
example, patients with limited disease, such as tracheobronchitis, who are diagnosed early and treated promptly, may have excellent outcomes. Conversely, liver transplant recipients, those with severe concomitant illness (e.g., dialysis dependence, hepatic insufficiency, malnutrition, mechanical ventilation or high transfusion requirements) and patients with dissemination to the CNS have much higher mortality rates [73,94,95].

The basic principles of therapy include effective antifungal therapy and reversal of immunosuppression as feasible. Timely initiation of effective antifungal treatment is likely essential for improving outcomes. Therefore, every effort should be made to establish the diagnosis as soon as possible, and empiric therapy should be strongly considered in cases where there is suspicion for invasive aspergillosis (e.g., subacute pulmonary processes, brain lesions). Even when the diagnosis is established and effective antifungal therapy is given, adjunctive surgical debridement is sometimes required. Length of therapy depends upon clinical and radiographic response, the patient's net state of immunosuppression and site and extent of infection. First-line treatment is with voriconazole [68]. Lipid formulations of AmB can be used as alternative agents in patients who cannot tolerate voriconazole, or whose disease progresses despite its use. However, initial therapy with an AmB preparation has been associated with increased risk of death in SOT recipients with invasive aspergillosis [95].

There is less experience with other agents. The role of echinocandins as monotherapy for invasive aspergillosis is unclear, but these agents are generally well tolerated and may be effective in selected patients [96]. Data regarding posaconazole in SOT patients with invasive aspergillosis are accumulating [97]. With the currently available formulation, steady state levels are not reached for several days after initiation of therapy, thereby limiting the utility of this drug as primary therapy. The main use of posaconazole for invasive aspergillosis in SOT patients at this time is as salvage therapy. The role of combination therapy for invasive aspergillosis remains unclear at this time. Treatment guidelines have not recommended routine use of primary combination therapy because clinical efficacy data have been lacking [68]. A recently completed clinical trial will hopefully shed light on this topic. Treatment with voriconazole and an echinocandin (e.g., caspofungin) may be beneficial for subsets of organ transplant recipients with invasive aspergillosis, such as those with renal failure, treatment failure with monotherapy and those with A. fumigatus infection [98].

Lung transplant recipients are at particularly high risk for development of invasive aspergillosis and should be considered for antifungal prophylaxis. Published guidelines recommend prophylaxis for those with Aspergillus colonization before or in the first year after transplantation, and those with two or more of the following: early airway ischemia, induction with alemtuzumab or thymoglobulin, single-lung transplant, CMV infection, rejection with augmented immunosuppression and acquired hypogammaglobulinemia (IgG $<400 \mathrm{mg} / \mathrm{dl}$ ) [99]. Prophylaxis approaches include inhaled AmB, itraconazole, and voriconazole [100]. The duration of antifungal prophylaxis depends on a dynamic assessment of risk factors. Most lung transplant centers employ universal prophylaxis with voriconazole alone or in combination with inhaled $\mathrm{AmB}$ in the first 6 months after transplant and many centers continue beyond that time [101]. 
The risk for invasive aspergillosis is significantly less for liver and heart transplant recipients. Antifungal prophylaxis is recommended for liver transplant recipients who are at risk for early invasive aspergillosis. These include patients receiving a liver retransplantation, and those with renal failure, particularly requiring renal replacement therapy. An additional risk is a reoperation involving the thoracic or abdominal cavity. Prophylaxis should include a lipid formulation of AmB or an echinocandin, and is continued for several weeks after transplant. Risk factors that should prompt consideration for prophylaxis in heart transplant recipients include isolation of Aspergillus species from respiratory tract cultures, reoperation, post-transplant hemodialysis and CMV disease. Voriconazole or itraconazole for several months after transplantation can be used [99].

\section{Cryptococcosis}

Cryptococcosis is the third most common cause of fungal infections in SOT recipients. It accounts for approximately $7-8 \%$ of fungal infections in this population. The majority of cases occur in kidney, and to a lesser extent, liver transplant recipients [1,2]. The infecting organism is generally $C$. neoformans, which has a worldwide distribution and is ubiquitous in the environment. In recent years, infections with Cryptococcus gattii have been increasingly reported in multiple locations in the USA, and in particular in the Pacific northwest region, and British Columbia in Canada. In a recent ana lysis of $C$. gattii cases, $20 \%$ of those patients from whom clinical details were available were SOT recipients [102].

Development of infection likely depends upon the interplay between the patient's net state of immunity and environmental exposure before or after transplantation. The time to onset of symptoms can be as short as 2-3 months after transplant, but the majority of cases occur between 2 and 5 years post-transplantation. Patients whose cryptococcosis is a result of reactivation of previous infection tend to develop disease earlier [103]. Risk factors for infection include corticosteroid use and receipt of multiple doses of T-cell depleting agents (e.g., antithymocyte globulin or alemtuzumab) [104]. Transmission of C. neoformans from a donor has been described in kidney, liver and lung transplant recipients $[5,14,18]$. Clues to such a transmission include onset of infection very early after transplantation and unusual sites of involvement such as the graft itself or the surgical site [105]. In a well-documented transmission event, three recipients contracted the infection from a common donor who had unexplained neurological symptoms at the time of his death. He was later discovered to have cryptococcal meningoencephalitis. All three developed cryptococcemia with either pneumonia or meningitis [18]. Differentiating donor-derived infection from reactivation of latent cryptococcosis may be difficult. Transplant centers should immediately notify the organ procurement agency if transmission is suspected, so that other recipients from a common donor can be notified.

The most common sites of infection are the lung and CNS. In approximately two-thirds of patients, the infection is disseminated to involve multiple organs [106]. The majority of patients with pulmonary cryptococcosis present with respiratory symptoms. Radiographic findings usually show single or multiple nodular lesions, or less commonly, pulmonary infiltrates [107]. Other manifestations include lung masses, cavities and pleural effusions. In some patients, pulmonary cryptococcosis is discovered incidentally when radiographic 
imaging is performed for another indication. The serum cryptococcal antigen may be a useful screening tool, but serum antigen tests are frequently negative in patients with lower organism burden, such as those with single nodules and lung transplant recipients with infection limited to the lung. Many patients with pulmonary cryptococcosis also have CNS disease, which can sometimes be asymptomatic. Therefore, all SOT recipients with pulmonary cryptococcosis should also be evaluated for CNS infection with a lumbar puncture.

CNS involvement is observed in nearly $50 \%$ of SOT recipients with cryptococcosis [108]. Clinical manifestations include headaches, changes in mental status, visual abnormalities and focal neurological findings. In addition to headaches and neurological abnormalities, clues to CNS involvement include late-onset disease ( $>2$ years after transplantation), serum cryptococcal antigen titers exceeding 1:64 and presence of fungemia [109]. Because calcineurin inhibitors possess a degree of anticryptococcal activity, immuno-compromised patients who are not receiving such drugs are at increased risk for CNS disease [110]. Infection can be limited to meningitis or involve the brain parenchyma. Outcomes tend to be worse with parenchymal compared with meningeal disease [111]. Diagnosis can be established with visualization of the fungus on Gram stain or India ink stain and by culture of cerebro-spinal fluid (CSF) fluid. However, direct staining may only be positive in 50\% and cultures in approximately $80 \%$ of those with CNS disease [111,112]. CSF cryptococcal antigen has excellent sensitivity and specificity (90\%).

A critical component of the evaluation is measurement of CSF pressure [113]. Multiple factors contribute to elevated CSF pressure in cryptococcosis, including inhibition of CSF absorption. Elevated pressures in cryptococcosis can lead to neurological injury that ranges in severity from altered mental status to visual and hearing loss and even death. When pressures exceed $25 \mathrm{cmH}_{2} \mathrm{O}$ of CSF, fluid should be removed to reduce intracranial pressure to $\angle 20 \mathrm{cmH}_{2} \mathrm{O}$. CSF taps should be repeated regularly until opening pressure remains below $25 \mathrm{cmH}_{2} \mathrm{O}$ [114]. In difficult to control cases, a lumbar drain or even a permanent ventriculoperitoneal shunt may need to be placed.

Besides the CNS and lung, cryptococcosis can involve almost any organ, including the skin, bones, joints, liver, kidney, prostate and the eye. Cutaneous cryptococcosis may be observed in nearly a fifth of cases in SOT recipients and typically manifests as nodules, masses, ulcers, abscesses and cellulitis. Skin involvement is usually observed as part of disseminated infection, but may be a lone finding and due to inoculation from an environmental source [115]. Cutaneous disease may be more common in certain regions such as the southern USA [116].

Overall survival in SOT patients with cryptococcosis is approximately 70-80\% [1,2]. Higher mortality rates have been associated with parenchymal brain lesions and in those with renal failure at baseline [106,111]. Receipt of a calcineurin inhibitor for immunosuppression may be associated with lower rates of mortality [106].

Antifungal therapy in cryptococcosis varies by site and extent of infection. In the case of neurological and/or severe pulmonary disease the treatment of choice is an AmB product. If 
possible, flucytosine should be added. Length of treatment with this initial therapy is usually 2 weeks, but ultimately depends upon clinical and micro-biological response to therapy. If flucytosine cannot be given, many authorities recommend extending AmB therapy to 4 weeks [114]. Once the patient has been stabilized with the above regimen, they can be transitioned to fluconazole at 400-800 mg daily for 8 more weeks and then 200-400 mg for 6-12 months. In cases of focal or incidentally detected pulmonary infection, fluconazole alone at $400 \mathrm{mg} / \mathrm{day}$ for 6-12 months may suffice. However, disseminated disease should be excluded with CSF ana lysis prior to embarking on this type of regimen.

An important aspect of anticryptococcosis therapy is reduction of immunosuppression. However, this should be done cautiously as too rapid a reduction may lead to an immune reconstitution syndrome (IRS). This complication occurs in 5-10\% of patients [117]. Manifestations include aseptic meningitis, cerebral mass lesions, spinal arachnoiditis and hydrocephalus. Other presentations include lymphadenitis, cellulitis or pulmonary nodules. Cultures are usually negative. IRS typically presents $4-6$ weeks after time of initiation of antifungal therapy and reduction of immunosuppression [118]. It may be associated with higher rates of allograft loss [119]. Some authorities recommend reduction as opposed to abrupt cessation of calcineurin inhibitors, with consideration given to tapering of corticosteroids first as a means of reducing immunosuppression without inducing IRS [120]. The optimal treatment of IRS is unclear. Corticosteroids may be considered in lifethreatening situations or severe disease. Emerging therapies include statins and tumor necrosis factor inhibitors, but at this time these should be considered experimental.

\section{Endemic mycoses}

H. capsulatum, Blastomyces dermatitidis and C. immitis are fungal pathogens with a limited geographic distribution [121]. Most reports of infections with these fungi are from patients who have resided in endemic areas. Collectively, they are referred to as the endemic fungi. In North America, H. capsulatum is endemic in multiple regions, but the most intense exposure is thought to occur in the Caribbean basin and in areas of the Mississippi and Ohio River Valleys. Inhalation of $H$. capsulatum can occur with exposure to disrupted soil around construction sites and agricultural areas where there are large concentrations of bird droppings. B. dermatitidis is endemic in areas of the Mississippi and Ohio River Valleys, the Great Lakes region and the St Lawrence Seaway. It is a rare cause of infection and typically affects immunocompetent men with outdoor occupations or pastimes. C. immitis is found in the dessert soil of northern Mexico, the southwest USA and areas of California's Central Valley. Exposure occurs when spores are aerosolized and inhaled.

Active disease may develop following environmental exposure, or due to reactivation of a latent infection acquired prior to transplantation. Most infections are diagnosed within the first year after transplantation and occur in kidney and liver transplant recipients [1]. Overall, approximately $2-5 \%$ of fungal infections in SOT recipients are attributed to endemic fungi $[1,2]$. However, this is likely an underestimation as a diagnosis is not always established in mild-to-moderate infections. Histoplasmosis accounts for the majority of cases and the infection is usually disseminated at time of diagnosis. However, the rates of 
infection vary by location. For example, in Arizona, USA, approximately 3-4\% of kidney and liver transplant recipients may develop coccidioidomycosis $[122,123]$.

Transmission of $H$. capsulatum via organ transplantation has been described. In one notable case, infection was transmitted by kidney donation to two patients. The donor, who was asymptomatic, had died from an unrelated cause and resided in an area heavily endemic for H. capsulatum. At 8 and 9 months after transplantation, the two recipients developed fever, weight loss, pancytopenia and $H$. capsulatum fungemia. Treatment with AmB was effective in both [12].

Prophylaxis with itraconazole may be appropriate for some patients. Radiographic evaluation of many potential donors from endemic areas may show signs of old, healed histoplasmosis. Findings include calcified pulmonary, hilar and splenic granulomata. However, radiographic evidence of quiescent or past infection are not considered a contraindication to donation [16]. Antifungal prophylaxis is recommended for lung transplant recipients whose donors have positive serology or incidental $H$. capsulatum detection in the donor lung [15].

Whether recipients of other organs from sero-positive donors should receive prophylaxis is controversial. Some authors recommend a course of at least 3-6 months, covering the period of more active immunosuppression [11]. Similarly, patients with history of histoplasmosis prior to their transplantation may benefit from prophylaxis. Such patients should have serial monitoring of urinary Histoplasma antigen during times of intense immunosuppression.

Transmission of coccidioidomycosis via organ transplantation has been also been described in lung, kidney and liver recipients. Most reports are from endemic areas or involved patients who had been former residents or visitors to those regions [7,10,11,124]. Published guidelines recommend antifungal prophylaxis if the donor had active Coccidioides infection or positive serologies [125]. Some authors recommend serological screening for coccidioidomycosis in donors from endemic areas to help guide prophylaxis [11]. A positive serology in the donor does not necessarily mandate rejection of the organ [15]. Patients with history of coccidioidomycosis or with positive Coccidioides serologies prior to their transplantation should receive fluconazole prophylaxis.

Clinical manifestations of endemic fungal infections can be varied and nonspecific. These may include fever, cough, shortness of breath and malaise. Nearly all patients have clinical or radiographic evidence of lung involvement [126]. The clinical course can be severe with high rates of disseminated disease and even death. Histoplasmosis has a predilection for organs of the reticuloendothelial system and infection can involve the bone marrow, lymphatic system, liver and spleen. Gastrointestinal mucosal ulceration, adrenal dysfunction, CNS involvement and fungemia may be observed with disseminated infection [127]. Blastomycosis may trigger acute respiratory distress syndrome and, when disseminated, can involve multiple organs including the skin, bones and spleen [128]. Coccidioidomycosis may disseminate to multiple sites including bones, joints, CNS and organs of the reticuloendothelial system $[123,129]$. 
Diagnosis can be made by identifying the organism on stain or culture from an affected site. Respiratory secretions, CSF, bloodstream, wound drainage and tissue specimen may all yield positive results. However, traditional microbiology and histopathology techniques often lack the sensitivity needed to detect infection. Additionally, appropriate specimens may be difficult to obtain. Nonculture-based techniques have greatly improved our diagnostic ability. In patients with disseminated histoplasmosis, testing of urine for Histoplasma antigen is highly sensitive and specific for infection. Sensitivity of the test declines with less severe infection and in immunocompetent patients [130]. Patients with more severe infection tend to have higher titers, which can facilitate measurement of the antigen to monitor response to therapy [131]. The urine antigen has substantial crossreactivity with $B$. dermatitidis infection, thus assisting in diagnosis of that infection. Recent evaluation of serum Histoplasma antigen suggests that this test performs well in patients with disseminated disease. Antibody detection may have a role in diagnosis of histoplasmosis, particularly in immuno competent patients with subacute infection. However, the sensitivity of such assays may be suboptimal in SOT recipients [130]. Serological testing can be useful in diagnosis of coccidioidomycosis. Often, numerous simultaneous methods are used for identifying coccidioidal antibodies, including enzyme immunoassay and immunodiffusion for IgM and IgG, and the complement fixation test. The sensitivity of these assays may be suboptimal in SOT recipients and a negative serological test does not necessarily rule out infection $[122,123]$.

Treatment varies by infecting pathogen and extent of infection. In cases of moderately severe and severe infection, the treatment of choice should be an AmB product. In general, AmB should be continued for approximately 2 weeks, but length of therapy depends upon the patient's response. Providing that the infection has been stabilized, the patient can be transitioned to oral fluconazole in the case of coccidioidomycosis and to oral itraconazole for histoplasmosis and blastomycosis. In cases of mild-to-moderate disease, the AmB portion of therapy can be omitted [125]. Total antifungal therapy course must be individualized, but in general, patients with blastomycosis and coccidioidomycosis should be treated for a minimum of 6-12 months and those with histoplasmosis for 12 months or longer.

\section{Rare filamentous fungi}

In recent years, dozens of fungal pathogens have been reported as rare causes of invasive fungal infections in SOT recipients. These include the Zygomycetes, which can cause potentially devastating infections that may complicate the course of SOT recipients [132134]. Risk factors for zygomycosis include uncontrolled diabetes mellitus, receipt of corticosteroids and neutropenia, all of which are common to many SOT recipients. Additional risk factors that have been described in SOT recipients include renal failure and prior voriconazole and/or caspofungin use [135]. Cases typically develop within 3-6 months of transplant, but may occur much later [136]. Other rare fungal infections may be due to hyaline molds (e.g., Fusarium and Scedosporium species), darkly pigmented molds and dimorphic fungal infections such as paracoccidioidomycosis and sporotrichosis [137]. Treatment options for emerging filamentous fungal pathogens have improved with the advent of newer mold-active azoles (e.g., voriconazole and posaconazole). However, 
susceptibility to these agents varies widely and data regarding ideal therapy in SOT recipients for many emerging fungi is scant. Moreover, an AmB product remains the drug of choice for treatment of zygomycosis and voriconazole lacks activity against the Zygomycetes.

\section{Conclusion \& future perspective}

Invasive fungal infections are an important cause of morbidity and mortality in SOT recipients. In the past few years there have been major advances in our understanding of the epidemiology of these infections and development of improved diagnostic and therapeutic tools. However, outcomes in patients with these infections continue to be suboptimal. In the next 5-10 years, several key trends will likely impact the landscape of invasive fungal infections occurring in SOT recipients. Specifically, these include a changing epidemiology of transplant organ recipients, an improved ability to diagnose fungal infections and widespread availability of newer antifungal agents. The epidemiology of organ recipients will be affected by use of novel and highly immunosuppressive regimens to prevent and manage organ rejection episodes. Moreover, mirroring national trends, the population of organ recipients will likely become increasingly diverse with respect to age, ethnicity and comorbid medical conditions. The ability to detect fungal colonization and/or infection will improve with further development and increasing deployment of nonculture-based diagnostic tools (e.g., antigen, antibody and nucleic acid detection assays). These, coupled with the increasing use of potent, broad-spectrum antifungal agents (e.g., voriconazole and posaconazole) for prophylaxis and treatment, will significantly alter the timing and outcomes of invasive fungal infections in this population. At the same time, advances in understanding fungal pathogenesis, host immune mechanisms and the pharmacology of antifungal compounds will provide new avenues for prevention and treatment. New data that integrates the results generated from traditional prospective clinical trials, clinical and genomic databases and laboratory-based investigations will be needed to meet these challenges.

\section{Acknowledgments}

S Shoham is on the advisory board and has acted as consultant for Merck, and has recieved research grants from Astellas, Merck and Pfizer. K Marr is on the advisory board and has acted as consultant for Astellas, Merck, Optimer and Pfizer, and has recieved research grants from Astellas, Merck and Pfizer. This work was supproted by the NIAID (K24 AI085118).

\section{References}

Papers of special note have been highlighted as:

of interest

- of considerable interest

1. Pappas PG, Alexander BD, Andes DR, et al. Invasive fungal infections among organ transplant recipients: results of the Transplant-Associated Infection Surveillance Network (TRANSNET). Clin. Infect. Dis. 2010; 50(8):1101-1111. [PubMed: 20218876] [Provides much-needed detail about the epidemiology of invasive fungal infections in solid organ transplant (SOT) recipients.] 
2. Neofytos D, Fishman JA, Horn D, et al. Epidemiology and outcome of invasive fungal infections in solid organ transplant recipients. Transplant. Infect. Dis. 2010; 12(3):220-229. [Provides much-needed detail about the epidemiology of invasive fungal infections in SOT recipients.]

3. Park BJ, Pappas PG, Wannemuehler KA, et al. Invasive non-aspergillus mold infections in transplant recipients, United States, 2001-2006. Emerg. Infect. Dis. 2011; 17(10):1855-1864. [PubMed: 22000355]

4. Fishman JA. Infection in solid-organ transplant recipients. N. Engl. J. Med. 2007; 357(25):26012614. [PubMed: 18094380]

5 . Ison MG, Hager J, Blumberg E, et al. Donor-derived disease transmission events in the United States: data reviewed by the OPTN/UNOS disease transmission advisory committee. Am. J. Transplant. 2009; 9(8):1929-1935. [PubMed: 19538493] [Summarizes cases of potential donorderived infections reported to the Organ Procurement and Transplantation Network from 2005 to 2007.]

6. van der Vliet JA, Tidow G, Kootstra G, et al. Transplantation of contaminated organs. Br. J. Surg. 1980; 67(8):596-598. [PubMed: 7000231]

7. Wright PW, Pappagianis D, Wilson M, et al. Donor-related coccidioidomycosis in organ transplant recipients. Clin. Infect. Dis. 2003; 37(9):1265-1269. [PubMed: 14557974]

8. Wong SY, Allen DM. Transmission of disseminated histoplasmosis via cadaveric renal transplantation: case report. Clin. Infect. Dis. 1992; 14(1):232-234. [PubMed: 1571437]

9. Mueller NJ, Weisser M, Fehr T, et al. Donor-derived aspergillosis from use of a solid organ recipient as a multiorgan donor. Transplant. Infect. Dis. 2010; 12(1):54-59.

10. Miller MB, Hendren R, Gilligan PH. Posttransplantation disseminated coccidioidomycosis acquired from donor lungs. J. Clin. Microbiol. 2004; 42(5):2347-2349. [PubMed: 15131231]

11. Martin-Davila P, Fortun J, Lopez-Velez R, et al. Transmission of tropical and geographically restricted infections during solid-organ transplantation. Clin. Microbiol. Rev. 2008; 21(1):60-96. [PubMed: 18202437]

12. Limaye AP, Connolly PA, Sagar M, et al. Transmission of histoplasma capsulatum by organ transplantation. N. Engl. J. Med. 2000; 343(16):1163-1166. [PubMed: 11036122]

13. Keating MR, Guerrero MA, Daly RC, Walker RC, Davies SF. Transmission of invasive aspergillosis from a subclinically infected donor to three different organ transplant recipients. Chest. 1996; 109(4):1119-1124. [PubMed: 8635345]

14. Kanj SS, Welty-Wolf K, Madden J, et al. Fungal infections in lung and heart-lung transplant recipients. Report of 9 cases and review of the literature. Med. (Baltimore). 1996; 75(3):142-156.

15. Grossi PA, Fishman JA. Donor-derived infections in solid organ transplant recipients. Am. J. Transplant. 2009; 9(Suppl. 4):S19-S26. [PubMed: 20070680]

16. Fischer SA, Avery RK. Screening of donor and recipient prior to solid organ transplantation. Am. J. Transplant. 2009; 9(Suppl. 4):S7-S18. [PubMed: 20070698]

17. Calvino J, Romero R, Pintos E, et al. Renal artery rupture secondary to pretransplantation Candida contamination of the graft in two different recipients. Am. J. Kidney Dis. 1999; 33(1):E3. [PubMed: 10074601]

18. Baddley JW, Schain DC, Gupte AA, et al. Transmission of Cryptococcus neoformans by organ transplantation. Clin. Infect. Dis. 2011; 52(4):E94-E98. [PubMed: 21220771]

19. Mattner F, Kola A, Fischer S, et al. Impact of bacterial and fungal donor organ contamination in lung, heart-lung, heart and liver transplantation. Infection. 2008; 36(3):207-212. [PubMed: 18470477]

20. Van Hal SJ, Marriott DJ, Chen SC, et al. Candidemia following solid organ transplantation in the era of antifungal prophylaxis: the Australian experience. Transplant. Infect. Dis. 2009; 11(2):122127.

21. Lockhart SR, Wagner D, Iqbal N, et al. Comparison of in vitro susceptibility characteristics of Candida species from cases of invasive candidiasis in solid organ and stem cell transplant recipients: Transplant-Associated Infections Surveillance Network (TRANSNET), 2001 to 2006. J. Clin. Microbiol. 2011; 49(7):2404-2410. [PubMed: 21562099] 
22. Tavanti A, Lupetti A, Ghelardi E, et al. Molecular monitoring of Candida albicans infections in liver transplant recipients. Eur. J. Clin. Microbiol. Infect. Dis. 2001; 20(8):544-553. [PubMed: 11681433]

23. Bendel CM, Wiesner SM, Garni RM, Cebelinski E, Wells CL. Cecal colonization and systemic spread of Candida albicans in mice treated with antibiotics and dexamethasone. Pediatr. Res. 2002; 51(3):290-295. [PubMed: 11861932]

24. Charles PE, Dalle F, Aube H, et al. Candida spp. colonization significance in critically ill medical patients: a prospective study. Intensive Care Med. 2005; 31(3):393-400. [PubMed: 15711782]

25. Bartholomew GA, Rodu B, Bell DS. Oral candidiasis in patients with diabetes mellitus: a thorough ana lysis. Diabetes Care. 1987; 10(5):607-612. [PubMed: 3677980]

26. Shoham S, Marwaha S. Invasive fungal infections in the ICU. J. Intensive Care Med. 2010; 25(2): 78-92. [PubMed: 19955115]

27. Nieto-Rodriguez JA, Kusne S, Manez R, et al. Factors associated with the development of candidemia and candidemia-related death among liver transplant recipients. Ann. Surg. 1996; 223(1):70-76. [PubMed: 8554421]

28. Patel R, Portela D, Badley AD, et al. Risk factors of invasive Candida and non-Candida fungal infections after liver transplantation. Transplantation. 1996; 62(7):926-934. [PubMed: 8878386]

29. Husain S, Tollemar J, Dominguez EA, et al. Changes in the spectrum and risk factors for invasive candidiasis in liver transplant recipients: prospective, multicenter, case-controlled study. Transplantation. 2003; 75(12):2023-2029. [PubMed: 12829905]

30. Alexander J, Limaye AP, Ko CW, Bronner MP, Kowdley KV. Association of hepatic iron overload with invasive fungal infection in liver transplant recipients. Liver Transplant. 2006; 12(12):1799-1804.

31. Pappas PG, Silveira FP. Candida in solid organ transplant recipients. Am. J. Transplant. 2009; 9(Suppl. 4):S173-S179. [PubMed: 20070678]

32. Kusne S, Furukawa H, Abu-Elmagd K, et al. Infectious complications after small bowel transplantation in adults: an update. Transplant. Proc. 1996; 28(5):2761-2762. [PubMed: 8908045]

33. Guaraldi G, Cocchi S, Codeluppi M, et al. Outcome, incidence, and timing of infectious complications in small bowel and multivisceral organ transplantation patients. Transplantation. 2005; 80(12):1742-1748. [PubMed: 16378070]

34. Battaglia M, Ditonno P, Selvaggio O, et al. Kidney transplants from infected donors: our experience. Transplant. Proc. 2004; 36(3):491-492. [PubMed: 15110567]

35. Albano L, Bretagne S, Mamzer-Bruneel MF, et al. Evidence that graft-site candidiasis after kidney transplantation is acquired during organ recovery: a multicenter study in France. Clin. Infect. Dis. 2009; 48(2):194-202. [PubMed: 19090753]

36. Mai H, Champion L, Ouali N, et al. Candida albicans arteritis transmitted by conservative liquid after renal transplantation: a report of four cases and review of the literature. Transplantation. 2006; 82(9):1163-1167. [PubMed: 17102767]

37. Gladdy RA, Richardson SE, Davies HD, Superina RA. Candida infection in pediatric liver transplant recipients. Liver Transplant. Surg. 1999; 5(1):16-24.

38. Florescu DF, Islam KM, Grant W, et al. Incidence and outcome of fungal infections in pediatric small bowel transplant recipients. Transplant. Infect. Dis. 2010; 12(6):497-504.

39. Reid GE, Grim SA, Sankary H, Benedetti E, Oberholzer J, Clark NM. Early intra-abdominal infections associated with orthotopic liver transplantation. Transplantation. 2009; 87(11):17061711. [PubMed: 19502964]

40. Said A, Safdar N, Lucey MR, et al. Infected bilomas in liver transplant recipients, incidence, risk factors and implications for prevention. Am. J. Transplant. 2004; 4(4):574-582. [PubMed: 15023150]

41. Safdar N, Said A, Lucey MR, et al. Infected bilomas in liver transplant recipients: clinical features, optimal management, and risk factors for mortality. Clin. Infect. Dis. 2004; 39(4):517-525. [PubMed: 15356815]

42. Nucci M, Anaissie E, Betts RF, et al. Early removal of central venous catheter in patients with candidemia does not improve outcome: ana lysis of 842 patients from 2 randomized clinical trials. Clin. Infect. Dis. 2010; 51(3):295-303. [PubMed: 20578829] 
43. Berenguer J, Buck M, Witebsky F, Stock F, Pizzo PA, Walsh TJ. Lysis-centrifugation blood cultures in the detection of tissue-proven invasive candidiasis. Disseminated versus single-organ infection. Diagn. Microbiol. Infect. Dis. 1993; 17(2):103-109. [PubMed: 8243032]

44. Avni T, Leibovici L, Paul M. PCR diagnosis of invasive candidiasis: systematic review and metaana lysis. J. Clin. Microbiol. 2011; 49(2):665-670. [PubMed: 21106797]

45. Ostrosky-Zeichner L. Invasive mycoses: diagnostic challenges. Am. J. Med. 2012; 125(Suppl. 1):S14-S24. [PubMed: 22196205]

46. Mennink-Kersten MA, Warris A, Verweij PE. 1,3- $\beta$-d-glucan in patients receiving intravenous amoxicillin-clavulanic acid. N. Engl. J. Med. 2006; 354(26):2834-2835. [PubMed: 16807428]

47. Mennink-Kersten MA, Ruegebrink D, Verweij PE. Pseudomonas aeruginosa as a cause of 1,3- $\beta$-dglucan assay reactivity. Clin. Infect. Dis. 2008; 46(12):1930-1931. [PubMed: 18540808]

48. Alexander BD, Smith PB, Davis RD, Perfect JR, Reller LB. The $(1,3)\{\beta\}$-d-glucan test as an aid to early diagnosis of invasive fungal infections following lung transplantation. J. Clin. Microbiol. 2010; 48(11):4083-4088. [PubMed: 20720025]

49. Chen J, Wang Y, Shen Z, Zhu Z, Song Y, Han R. Early diagnostic value of plasma PCT and BG assay for CRBSI after OLT. Transplant. Proc. 2011; 43(5):1777-1779. [PubMed: 21693277]

50. Pappas PG, Kauffman CA, Andes D, et al. Clinical practice guidelines for the management of candidiasis: 2009 update by the Infectious Diseases Society of America. Clin. Infect. Dis. 2009; 48(5):503-535. [PubMed: 19191635]

51. Wahidi MM, Willner DA, Snyder LD, Hardison JL, Chia JY, Palmer SM. Diagnosis and outcome of early pleural space infection following lung transplantation. Chest. 2009; 135(2):484-491. [PubMed: 19017896]

52. Kovats Z, Sutto Z, Murakozy G, et al. Airway pathogens during the first year after lung transplantation: a single-center experience. Transplant. Proc. 2011; 43(4):1290-1291. [PubMed: 21620113]

53. Schaenman JM, Rosso F, Austin JM, et al. Trends in invasive disease due to Candida species following heart and lung transplantation. Transplant. Infect. Dis. 2009; 11(2):112-121.

54. Horvath J, Dummer S, Loyd J, Walker B, Merrill WH, Frist WH. Infection in the transplanted and native lung after single lung transplantation. Chest. 1993; 104(3):681-685. [PubMed: 8365275]

55. Kubak BM. Fungal infection in lung transplantation. Transplant. Infect. Dis. 2002; 4(Suppl. 3 ):24 31.

56. Hadjiliadis D, Howell DN, Davis RD, et al. Anastomotic infections in lung transplant recipients. Ann. Transplant. 2000; 5(3):13-19. [PubMed: 11147024]

57. Delgado J, Calvo N, Gomis A, et al. Candiduria in renal transplant recipients: incidence, clinical repercussion, and treatment indication. Transplant. Proc. 2010; 42(8):2944-2946. [PubMed: 20970578]

58. Safdar N, Slattery WR, Knasinski V, et al. Predictors and outcomes of candiduria in renal transplant recipients. Clin. Infect. Dis. 2005; 40(10):1413-1421. [PubMed: 15844063] [Indicated that candiduria was associated with reduced survival rates among renal transplant recipients, but that treatment of asymptomatic candiduria did not improve outcomes.]

59. Sobel JD, Bradshaw SK, Lipka CJ, Kartsonis NA. Caspofungin in the treatment of symptomatic candiduria. Clin. Infect. Dis. 2007; 44(5):E46-E49. [PubMed: 17278048]

60. Ostrosky-Zeichner L, Pappas PG, Shoham S, et al. Improvement of a clinical prediction rule for clinical trials on prophylaxis for invasive candidiasis in the intensive care unit. Mycoses. 2011; 54(1):46-51. [PubMed: 19627509]

61. Hermsen ED, Zapapas MK, Maiefski M, Rupp ME, Freifeld AG, Kalil AC. Validation and comparison of clinical prediction rules for invasive candidiasis in intensive care unit patients: a matched case-control study. Crit. Care. 2011; 15(4):R198. [PubMed: 21846332]

62. Senn L, Eggimann P, Ksontini R, et al. Caspofungin for prevention of intra-abdominal candidiasis in high-risk surgical patients. Intensive Care Med. 2009; 35(5):903-908. [PubMed: 19172247]

63. Fortun J, Martin-Davila P, Montejo M, et al. Prophylaxis with caspofungin for invasive fungal infections in high-risk liver transplant recipients. Transplantation. 2009; 87(3):424-435. [PubMed: 19202450] 
64. Castroagudin JF, Ponton C, Bustamante M, et al. Prospective interventional study to evaluate the efficacy and safety of liposomal amphotericin B as prophylaxis of fungal infections in high-risk liver transplant recipients. Transplant. Proc. 2005; 37(9):3965-3967. [PubMed: 16386598]

65. Singhal S, Ellis RW, Jones SG, et al. Targeted prophylaxis with amphotericin B lipid complex in liver transplantation. Liver Transplant. 2000; 6(5):588-595.

66. Winston DJ, Pakrasi A, Busuttil RW. Prophylactic fluconazole in liver transplant recipients. A randomized, double-blind, placebo-controlled trial. Ann. Intern. Med. 1999; 131(10):729-737. [PubMed: 10577295] [Established the role of fluconazole prophylaxis in liver transplant recipients.]

67. Morgan J, Wannemuehler KA, Marr KA, et al. Incidence of invasive aspergillosis following hematopoietic stem cell and solid organ transplantation: interim results of a prospective multicenter surveillance program. Med. Mycol. 2005; 43(Suppl. 1):S49-S58. [PubMed: 16110792]

68. Walsh TJ, Anaissie EJ, Denning DW, et al. Treatment of aspergillosis: clinical practice guidelines of the Infectious Diseases Society of America. Clin. Infect. Dis. 2008; 46(3):327-360. [PubMed: 18177225]

69. Panackal AA, Dahlman A, Keil KT, et al. Outbreak of invasive aspergillosis among renal transplant recipients. Transplantation. 2003; 75(7):1050-1053. [PubMed: 12698098]

70. Raviv Y, Kramer MR, Amital A, Rubinovitch B, Bishara J, Shitrit D. Outbreak of aspergillosis infections among lung transplant recipients. Transplant. Int. 2007; 20(2):135-140.

71. Pegues CF, Daar ES, Murthy AR. The epidemiology of invasive pulmonary aspergillosis at a large teaching hospital. Infect. Control Hosp. Epidemiol. 2001; 22(6):370-374. [PubMed: 11519915]

72. Pegues DA, Lasker BA, Mcneil MM, Hamm PM, Lundal JL, Kubak BM. Cluster of cases of invasive aspergillosis in a transplant intensive care unit: evidence of person-to-person airborne transmission. Clin. Infect. Dis. 2002; 34(3):412-416. [PubMed: 11753826]

73. Gavalda J, Len O, San Juan R, et al. Risk factors for invasive aspergillosis in solid-organ transplant recipients: a case-control study. Clin. Infect. Dis. 2005; 41(1):52-59. [PubMed: 15937763]

74. Rosenhagen M, Feldhues R, Schmidt J, Hoppe-Tichy T, Geiss HK. A risk profile for invasive aspergillosis in liver transplant recipients. Infection. 2009; 37(4):313-319. [PubMed: 19629387]

75. Singh N, Pruett TL, Houston S, et al. Invasive aspergillosis in the recipients of liver retransplantation. Liver Transplant. 2006; 12(8):1205-1209.

76. Fortun J, Martin-Davila P, Moreno S, et al. Risk factors for invasive aspergillosis in liver transplant recipients. Liver Transplant. 2002; 8(11):1065-1070.

77. Weigt SS, Elashoff RM, Huang C, et al. Aspergillus colonization of the lung allograft is a risk factor for bronchiolitis obliterans syndrome. Am. J. Transplant. 2009; 9(8):1903-1911. [PubMed: 19459819]

78. Goldfarb NS, Avery RK, Goormastic M, et al. Hypogammaglobulinemia in lung transplant recipients. Transplantation. 2001; 71(2):242-246. [PubMed: 11213067]

79. Husni RN, Gordon SM, Longworth DL, et al. Cytomegalovirus infection is a risk factor for invasive aspergillosis in lung transplant recipients. Clin. Infect. Dis. 1998; 26(3):753-755. [PubMed: 9524855]

80. Westney GE, Kesten S, De Hoyos A, Chapparro C, Winton T, Maurer JR. Aspergillus infection in single and double lung transplant recipients. Transplantation. 1996; 61(6):915-919. [PubMed: 8623160]

81. Grossi P, Farina C, Fiocchi R, Dalla Gasperina D. Prevalence and outcome of invasive fungal infections in 1,963 thoracic organ transplant recipients: a multicenter retrospective study. Italian study group of fungal infections in thoracic organ transplant recipients. Transplantation. 2000; 70(1):112-116. [PubMed: 10919584]

82. Ersoy A, Dizdar OS, Koc AO, Akalin H, Ener B. Aspergillus fumigatus spondylodiskitis in renal transplant patient: voriconazole experience. Exp. Clin. Transplant. 2011; 9(4):265-269. [PubMed: 21819372]

83. Taillandier J, Alemanni M, Cerrina J, Le Roy Ladurie F, Dartevelle P. Aspergillus osteomyelitis after heart-lung transplantation. J. Heart Lung Transplant. 1997; 16(4):436-438. [PubMed: 9154954] 
84. Hope WW, Walsh TJ, Denning DW. Laboratory diagnosis of invasive aspergillosis. Lancet Infect. Dis. 2005; 5(10):609-622. [PubMed: 16183515]

85. Park YS, Seo JB, Lee YK, et al. Radiological and clinical findings of pulmonary aspergillosis following solid organ transplant. Clin. Radiol. 2008; 63(6):673-680. [PubMed: 18455559]

86. Pfeiffer CD, Fine JP, Safdar N. Diagnosis of invasive aspergillosis using a galactomannan assay: a meta-ana lysis. Clin. Infect. Dis. 2006; 42(10):1417-1427. [PubMed: 16619154]

87. Clancy CJ, Jaber RA, Leather HL, et al. Bronchoalveolar lavage galactomannan in diagnosis of invasive pulmonary aspergillosis among solid-organ transplant recipients. J. Clin. Microbiol. 2007; 45(6):1759-1765. [PubMed: 17428933]

88 . Luong ML, Clancy CJ, Vadnerkar A, et al. Comparison of an aspergillus real-time polymerase chain reaction assay with galactomannan testing of bronchoalvelolar lavage fluid for the diagnosis of invasive pulmonary aspergillosis in lung transplant recipients. Clin. Infect. Dis. 2011; 52(10):1218-1226. [PubMed: 21507918] [Investigated the utility of two nonculture-based diagnostic assays, alone and in combination, for the detection of invasive pulmonary aspergillosis.]

89. Pasqualotto AC, Xavier MO, Sanchez LB, et al. Diagnosis of invasive aspergillosis in lung transplant recipients by detection of galactomannan in the bronchoalveolar lavage fluid. Transplantation. 2010; 90(3):306-311. [PubMed: 20548265]

90. Husain S, Paterson DL, Studer SM, et al. Aspergillus galactomannan antigen in the bronchoalveolar lavage fluid for the diagnosis of invasive aspergillosis in lung transplant recipients. Transplantation. 2007; 83(10):1330-1336. [PubMed: 17519782]

91. Husain S, Clancy CJ, Nguyen MH, et al. Performance characteristics of the platelia Aspergillus enzyme immunoassay for detection of Aspergillus galactomannan antigen in bronchoalveolar lavage fluid. Clin. Vaccin. Immunol. 2008; 15(12):1760-1763.

92. Boonsarngsuk V, Niyompattama A, Teosirimongkol C, Sriwanichrak K. False-positive serum and bronchoalveolar lavage Aspergillus galactomannan assays caused by different antibiotics. Scand. J. Infect. Dis. 2010; 42((6-7):461-468. [PubMed: 20192889]

93. Fortun J, Martin-Davila P, Alvarez ME, et al. False-positive results of Aspergillus galactomannan antigenemia in liver transplant recipients. Transplantation. 2009; 87(2):256-260. [PubMed: 19155981]

94. Cornet M, Fleury L, Maslo C, Bernard JF, Brucker G. Epidemiology of invasive aspergillosis in France: a six-year multicentric survey in the Greater Paris area. J. Hosp. Infect. 2002; 51(4):288296. [PubMed: 12183144]

95. Baddley JW, Andes DR, Marr KA, et al. Factors associated with mortality in transplant patients with invasive aspergillosis. Clin. Infect. Dis. 2010; 50(12):1559-1567. [PubMed: 20450350]

96. Petrovic J, Ngai A, Bradshaw S, et al. Efficacy and safety of caspofungin in solid organ transplant recipients. Transplant. Proc. 2007; 39(10):3117-3120. [PubMed: 18089335]

97. Alexander BD, Perfect JR, Daly JS, et al. Posaconazole as salvage therapy in patients with invasive fungal infections after solid organ transplant. Transplantation. 2008; 86(6):791-796. [PubMed: 18813103]

98. Singh N, Limaye AP, Forrest G, et al. Combination of voriconazole and caspofungin as primary therapy for invasive aspergillosis in solid organ transplant recipients: a prospective, multicenter, observational study. Transplantation. 2006; 81(3):320-326. [PubMed: 16477215]

99. Singh N, Husain S. Invasive aspergillosis in solid organ transplant recipients. Am. J. Transplant. 2009; 9(Suppl. 4):S180-S191. [PubMed: 20070679]

100. Cadena J, Levine DJ, Angel LF, et al. Antifungal prophylaxis with voriconazole or itraconazole in lung transplant recipients: hepatotoxicity and effectiveness. Am. J. Transplant. 2009; 9(9):20852091. [PubMed: 19645709]

101. Neoh CF, Snell GI, Kotsimbos T, et al. Antifungal prophylaxis in lung transplantation - a worldwide survey. Am. J. Transplant. 2011; 11(2):361-366. [PubMed: 21272239]

102. Harris JR, Lockhart SR, Debess E, et al. Cryptococcus gattii in the United States: clinical aspects of infection with an emerging pathogen. Clin. Infect. Dis. 2011; 53(12):1188-1195. [PubMed: 22016503] 
103. Saha DC, Goldman DL, Shao X, et al. Serologic evidence for reactivation of cryptococcosis in solid-organ transplant recipients. Clin. Vaccin. Immunol. 2007; 14(12):1550-1554.

104. Silveira FP, Husain S, Kwak EJ, et al. Cryptococcosis in liver and kidney transplant recipients receiving anti-thymocyte globulin or alemtuzumab. Transplant. Infect. Dis. 2007; 9(1):22-27.

105. Sun HY, Alexander BD, Lortholary O, et al. Unrecognized pretransplant and donor-derived cryptococcal disease in organ transplant recipients. Clin. Infect. Dis. 2010; 51(9):1062-1069. [PubMed: 20879857]

106. Singh N, Alexander BD, Lortholary O, et al. Cryptococcus neoformans in organ transplant recipients: impact of calcineurin-inhibitor agents on mortality. J. Infect. Dis. 2007; 195(5):756764. [PubMed: 17262720]

107. Singh N, Alexander BD, Lortholary O, et al. Pulmonary cryptococcosis in solid organ transplant recipients: clinical relevance of serum cryptococcal antigen. Clin. Infect. Dis. 2008; 46(2):e12e18. [PubMed: 18171241]

108. Davis JA, Horn DL, Marr KA, Fishman JA. Central nervous system involvement in cryptococcal infection in individuals after solid organ transplantation or with AIDS. Transplant. Infect. Dis. 2009; 11(5):432-437.

109. Osawa R, Alexander BD, Lortholary O, et al. Identifying predictors of central nervous system disease in solid organ transplant recipients with cryptococcosis. Transplantation. 2010; 89(1):6974. [PubMed: 20061921]

110. Husain S, Wagener MM, Singh N. Cryptococcus neoformans infection in organ transplant recipients: variables influencing clinical characteristics and outcome. Emerg. Infect. Dis. 2001; 7(3):375-381. [PubMed: 11384512]

111. Singh N, Lortholary O, Dromer F, et al. Central nervous system cryptococcosis in solid organ transplant recipients: clinical relevance of abnormal neuroimaging findings. Transplantation. 2008; 86(5):647-651. [PubMed: 18791444]

112. Wu G, Vilchez RA, Eidelman B, Fung J, Kormos R, Kusne S. Cryptococcal meningitis: an ana lysis among 5,521 consecutive organ transplant recipients. Transplant. Infect. Dis. 2002; 4(4): $183-188$.

113. Shoham S, Cover C, Donegan N, Fulnecky E, Kumar P. Cryptococcus neoformans meningitis at 2 hospitals in Washington, D.C.: adherence of health care providers to published practice guidelines for the management of cryptococcal disease. Clin. Infect. Dis. 2005; 40(3):477-479. [PubMed: 15668874]

114. Singh N, Forrest G. Cryptococcosis in solid organ transplant recipients. Am. J. Transplant. 2009; 9(Suppl. 4):S192-S198. [PubMed: 20070681]

115. Sun HY, Alexander BD, Lortholary O, et al. Cutaneous cryptococcosis in solid organ transplant recipients. Med. Mycol. 2010; 48(6):785-791. [PubMed: 20100136]

116. Osawa R, Alexander BD, Forrest GN, et al. Geographic differences in disease expression of cryptococcosis in solid organ transplant recipients in the United States. Ann. Transplant. 2010; 15(4):77-83. [PubMed: 21183881]

117. Sun HY, Singh N. Opportunistic infection-associated immune reconstitution syndrome in transplant recipients. Clin. Infect. Dis. 2011; 53(2):168-176. [PubMed: 21690625]

118. Singh N, Lortholary O, Alexander BD, et al. An immune reconstitution syndrome-like illness associated with Cryptococcus neoformans infection in organ transplant recipients. Clin. Infect. Dis. 2005; 40(12):1756-1761. [PubMed: 15909263]

119. Singh N, Lortholary O, Alexander BD, et al. Allograft loss in renal transplant recipients with Cryptococcus neoformans associated immune reconstitution syndrome. Transplantation. 2005; 80(8):1131-1133. [PubMed: 16278598]

120. Singh N. How I treat cryptococcosis in organ transplant recipients. Transplantation. 2012; 93(1): 17-21. [PubMed: 22015465]

121. Baddley JW, Winthrop KL, Patkar NM, et al. Geographic distribution of endemic fungal infections among older persons, United States. Emerg. Infect. Dis. 2011; 17(9):1664-1669. [PubMed: 21888792]

122. Vucicevic D, Carey EJ, Blair JE. Coccidioidomycosis in liver transplant recipients in an endemic area. Am. J. Transplant. 2011; 11(1):111-119. [PubMed: 21087416] 
123. Braddy CM, Heilman RL, Blair JE. Coccidioidomycosis after renal transplantation in an endemic area. Am. J. Transplant. 2006; 6(2):340-345. [PubMed: 16426318]

124. Tripathy U, Yung GL, Kriett JM, Thistlethwaite PA, Kapelanski DP, Jamieson SW. Donor transfer of pulmonary coccidioidomycosis in lung transplantation. Ann. Thorac. Surg. 2002; 73(1):306-308. [PubMed: 11834038]

125. Proia L, Miller R. Endemic fungal infections in solid organ transplant recipients. Am. J. Transplant. 2009; 9(Suppl. 4):S199-S207. [PubMed: 20070682]

126. Cuellar-Rodriguez J, Avery RK, Lard M, et al. Histoplasmosis in solid organ transplant recipients: 10 years of experience at a large transplant center in an endemic area. Clin. Infect. Dis. 2009; 49(5):710-716. [PubMed: 19635026]

127. Grim SA, Proia L, Miller R, et al. A multicenter study of histoplasmosis and blastomycosis after solid organ transplantation. Transplant. Infect. Dis. 2011; 14(1):17-23.

128. Gauthier GM, Safdar N, Klein BS, Andes DR. Blastomycosis in solid organ transplant recipients. Transplant. Infect. Dis. 2007; 9(4):310-317.

129. Blair JE, Logan JL. Coccidioidomycosis in solid organ transplantation. Clin. Infect. Dis. 2001; 33(9):1536-1544. [PubMed: 11588699]

130. Hage CA, Ribes JA, Wengenack NL, et al. A multicenter evaluation of tests for diagnosis of histoplasmosis. Clin. Infect. Dis. 2011; 53(5):448-454. [PubMed: 21810734]

131. Wheat LJ, Freifeld AG, Kleiman MB, et al. Clinical practice guidelines for the management of patients with histoplasmosis: 2007 update by the Infectious Diseases Society of America. Clin. Infect. Dis. 2007; 45(7):807-825. [PubMed: 17806045]

132. Jimenez C, Lumbreras C, Aguado JM, et al. Successful treatment of mucor infection after liver or pancreas-kidney transplantation. Transplantation. 2002; 73(3):476-480. [PubMed: 11884949]

133. Singh N, Gayowski T, Singh J, Yu VL. Invasive gastrointestinal zygomycosis in a liver transplant recipient: case report and review of zygomycosis in solid-organ transplant recipients. Clin. Infect. Dis. 1995; 20(3):617-620. [PubMed: 7756485]

134. Sun HY, Forrest G, Gupta KL, et al. Rhino-orbital-cerebral zygomycosis in solid organ transplant recipients. Transplantation. 2010; 90(1):85-92. [PubMed: 20626095]

135. Singh N, Aguado JM, Bonatti H, et al. Zygomycosis in solid organ transplant recipients: a prospective, matched case-control study to assess risks for disease and outcome. J. Infect. Dis. 2009; 200(6):1002-1011. [PubMed: 19659439]

136. Sun HY, Aguado JM, Bonatti H, et al. Pulmonary zygomycosis in solid organ transplant recipients in the current era. Am. J. Transplant. 2009; 9(9):2166-2171. [PubMed: 19681829]

137. Kubak BM, Huprikar SS. Emerging \& rare fungal infections in solid organ transplant recipients. Am. J. Transplant. 2009; 9(Suppl. 4):S208-S226. [PubMed: 20070683]

138. Perfect JR, Dismukes WE, Dromer F, et al. Clinical practice guidelines for the management of cryptococcal disease: 2010 update by the infectious diseases society of america. Clin. Infect. Dis. 2010; 50(3):291-322. [PubMed: 20047480]

139. Galgiani JN, Catanzaro A, Cloud GA, et al. Comparison of oral fluconazole and itraconazole for progressive, nonmeningeal coccidioidomycosis. A randomized, double-blind trial. Mycoses study group. Ann. Intern. Med. 2000; 133(9):676-686. [PubMed: 11074900]

140. Walsh TJ, Raad I, Patterson TF, et al. Treatment of invasive aspergillosis with posaconazole in patients who are refractory to or intolerant of conventional therapy: an externally controlled trial. Clin. Infect. Dis. 2007; 44(1):2-12. [PubMed: 17143808]

141. Calvo E, Pastor FJ, Salas V, Mayayo E, Capilla J, Guarro J. Histopathology and antifungal treatment of experimental murine chromoblastomycosis caused by Cladophialophora carrionii. J. Antimicrob. Chemother. 2012; 67(3):666-670. [PubMed: 22190608]

142 . Herbrecht R, Denning DW, Patterson TF, et al. Voriconazole versus amphotericin B for primary therapy of invasive aspergillosis. N. Engl. J. Med. 2002; 347(6):408-415. [PubMed: 12167683] [Challenged the supremacy of amphotericin B, and established voriconazole as a firstline agent for invasive aspergillosis.]

143. Ostrosky-Zeichner L, Oude Lashof AM, Kullberg BJ, Rex JH. Voriconazole salvage treatment of invasive candidiasis. Eur. J. Clin. Microbiol. Infect. Dis. 2003; 22(11):651-655. [PubMed: 14564539] 
144. Roden MM, Zaoutis TE, Buchanan WL, et al. Epidemiology and outcome of zygomycosis: a review of 929 reported cases. Clin. Infect. Dis. 2005; 41(5):634-653. [PubMed: 16080086]

145ם. Reboli AC, Rotstein C, Pappas PG, et al. Anidulafungin versus fluconazole for invasive candidiasis. N. Engl. J. Med. 2007; 356(24):2472-2482. [PubMed: 17568028] [Indicated that echinocandins may be the most effective antifungal agent for the initial treatment of invasive candidiasis.]

146. Walsh TJ, Goutelle S, Jelliffe RW, et al. Intrapulmonary pharmacokinetics and pharmacodynamics of micafungin in adult lung transplant patients. Antimicrob. Agents Chemother. 2010; 54(8):3451-3459. [PubMed: 20439610]

147. Maertens J, Raad I, Petrikkos G, et al. Efficacy and safety of caspofungin for treatment of invasive aspergillosis in patients refractory to or intolerant of conventional antifungal therapy. Clin. Infect. Dis. 2004; 39(11):1563-1571. [PubMed: 15578352]

148. Winkler M, Pratschke J, Schulz U, et al. Caspofungin for post solid organ transplant invasive fungal disease: results of a retrospective observational study. Transplant. Infect. Dis. 2010; 12(3): 230-237.

149. Brouwer AE, Rajanuwong A, Chierakul W, et al. Combination antifungal therapies for HIVassociated cryptococcal meningitis: a randomised trial. Lancet. 2004; 363(9423):1764-1767. [PubMed: 15172774]

150. Shoham, S. Antifungal agents in adult transplant recipients.. In: Kumar, D.; Humar, A., editors. The AST Handbook of Transplant Infections. Wiley-Blackwell; UK: 2011. p. 146-149. 


\section{Executive summary}

\section{General epidemiology}

The major factors involved in development of invasive fungal infections are the patient's net state of immunosuppression, contact with pathogenic fungi (due to colonization or from environmental exposure) and use of antifungal prophylaxis.

The most common invasive fungal infections in solid organ transplant (SOT) recipients (except for lung transplant) are candidiasis followed by aspergillosis and cryptococcosis. Aspergillosis is the most common in lung transplant recipients.

\section{Candidiasis}

Candida is usually a harmless colonizer of the skin, GI tract and female genitourinary tract.

Invasive candidiasis is the most common fungal infection in SOT recipients.

Invasive candidiasis is associated with factors that increase yeast burden (e.g., antibacterial antibiotics, hyperglycemia), disrupt anatomical barriers (e.g., gastrointestinal mucosal injury, indwelling vascular devices) and impair neutrophil function and number (e.g., cytotoxic chemotherapy, corticosteroids).

Traditional culture techniques are often insensitive, and therefore newer diagnostic assays are desperately needed, and therapy is often empiric.

\section{Aspergillosis}

Aspergillus is ubiquitous in the environment and a frequent colonizer of chronically diseased airways (e.g., in patients with cystic fibrosis).

Invasive aspergillosis is the most common filamentous fungal infection in SOT recipients. Risk factors include neutropenia, neutrophil dysfunction (particularly with corticosteroids) and chronic lung disease.

Diagnosis typically relies on staining, culture and histopathology of relevant specimen. Because these are often insensitive or difficult to acquire (e.g., lung biopsy), newer assays, particularly serum and bronchoalveolar lavage galactomannan measurements have emerged as important alternatives.

\section{Cryptococcosis}

Cryptococcus neoformans is ubiquitous in the environment.

Infection typically involves the lungs and/or CNS. Cryptococcosis involving the CNS may have very few symptoms initially, but can lead to devastating neurological consequences if untreated.

Diagnosis is by culture and detection of cryptococcal antigen in cerebrospinal fluid and serum. Cerebrospinal fluid pressure management is an important component of CNS disease.

The length of treatment may be months to years. 


\section{Endemic mycoses}

Histoplasma capsulatum and Coccidioides immitis are limited to specific geographic regions.

Infection typically involves the lungs, but can disseminate to organs of the reticuloendothelial system (histoplasmosis), skin and bone (coccidioidomycosis) and CNS (both).

In addition to culture and histopathology, Histoplasma antigen and serology, and Coccidioides serology can assist with diagnosis.

The length of treatment may be months to years. 


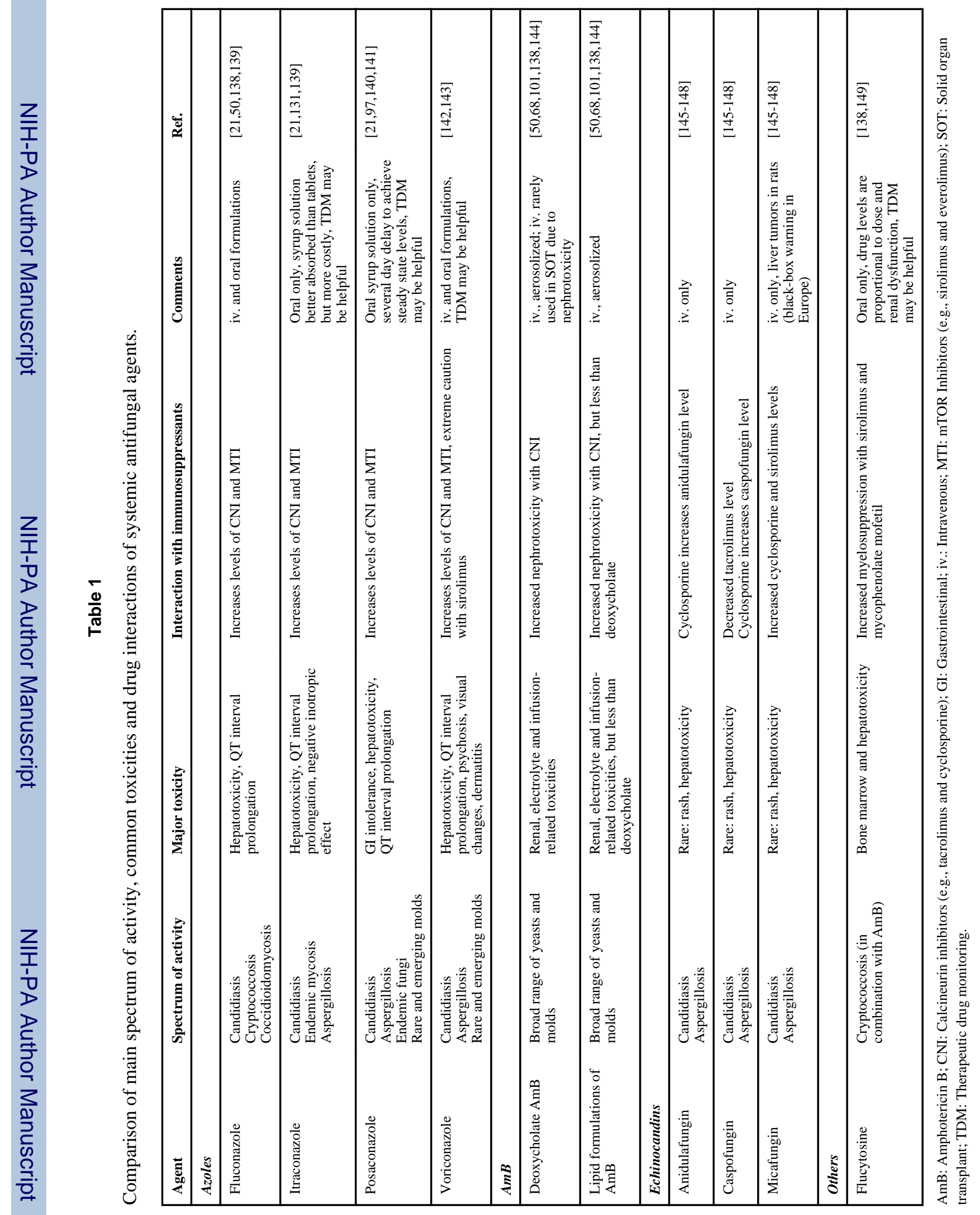

Future Microbiol. Author manuscript; available in PMC 2014 November 06. 
